\title{
A review on the possible existence of strong elementary charge and its nuclear scale applications
}

\author{
U. V. S. Seshavatharam ${ }^{1} \&$ S. Lakshminarayana ${ }^{2}$ \\ ${ }^{1}$ Honorary Faculty, I-SERVE, Survey no-42, Hitech city, Hyderabad-84, Telangana, India. \\ ${ }^{2}$ Department of Nuclear Physics, Andhra University, Visakhapatnam-03, AP, India \\ Emails: seshavatharam.uvs@gmail.com and sln@auvsp.edu.in
}

\begin{abstract}
We review the basics of nuclear binding energy scheme assumed to be associated with the existence of a new strong elementary charge associated with square root of reciprocal of the strong coupling constant.
\end{abstract}

Keywords: strong coupling constant, strong elementary charge, nuclear binding energy.

\section{Introduction}

As strong interaction [1] is mostly hidden at low energy scales in the form of 'residual nuclear force' and Liquid drop model and Fermi gas model [2-5] are failing in understanding nuclear binding energy with 'strong coupling constant', in our earlier published paper [6] and recent submitted papers $[7,8]$ we suggested that, by considering 'square root' of reciprocal of the strong coupling constant' $\left(\alpha_{s} \cong 0.1186\right)$, as an index of strength of nuclear elementary charge, nuclear binding energy and nuclear stability can be understood. Our model [6-11] seems to be simple and realistic compared to the new integrated model $[12,13]$. In this paper we review sections 6 and 7 with much better semi empirical relations.

\section{About the semi empirical mass formula}

Let $A$ be the total number of nucleons, $Z$ the number of protons and $N$ the number of neutrons. According to the semi-empirical mass formula $[2,3,4]$, nuclear binding energy:

$$
B \cong a_{v} A-a_{s} A^{2 / 3}-a_{c} \frac{Z(Z-1)}{A^{1 / 3}}-a_{a} \frac{(A-2 Z)^{2}}{A} \pm \frac{a_{p}}{\sqrt{A}}
$$

Here $a_{v}=$ volume energy coefficient, $a_{s}$ is the surface energy coefficient, $a_{c}$ is the coulomb energy coefficient, $a_{a}$ is the asymmetry energy coefficient and $a_{p}$ is the pairing energy coefficient. By maximizing $B(A, Z)$ with respect to $Z$,

$$
Z \approx \frac{A}{2+\left(a_{c} / 2 a_{a}\right) A^{2 / 3}} \text { and } A-2 Z \approx \frac{0.4 A^{2}}{A+200}
$$

Maximizing $B(A) / A$ with respect to $A$ gives the nucleus which is most strongly bound or most stable.

\section{New concepts and semi empirical relations of nuclear binding energy and stability}

We would like to suggest that,

1) There exists a strong nuclear charge, $e_{s} \cong \frac{e}{\sqrt{\alpha_{s}}} \cong 4.6523 \times 10^{-19} \mathrm{C}$

2) Proton magnetic moment [1] can be addressed with $\mu_{p} \cong \frac{e_{s} \hbar}{2 m_{p}} \cong 1.467 \times 10^{-26} \mathrm{~J}^{-1}$

3) Neutron magnetic moment [1] can be addressed with $\mu_{n} \cong\left(e_{s}-e\right) \frac{\hbar}{2 m_{n}} \cong 9.602 \times 10^{-27}{\mathrm{~J} . \mathrm{T}^{-1}}^{-}$

4) Characteristic nuclear radius can be expressed as, 


$$
R_{0} \cong\left(\frac{1}{\sqrt{\alpha_{s}}}\right)\left\{\frac{\hbar}{m_{p} c}+\frac{\hbar}{m_{n} c}\right\} \cong\left(\frac{e_{s}}{e}\right)\left(\frac{2 \hbar}{m_{p} c}\right) \cong 1.22 \mathrm{fm} .
$$

where $m_{p} \approx m_{n}$.

5) Nuclear beta stability line [4] can be addressed with a relation of the form, $A_{s} \cong 2 Z+s(2 Z)^{2}$ where $s \cong\left\{\left(\frac{e_{s}}{m_{p}}\right) \div\left(\frac{e}{m_{e}}\right)\right\} \cong 0.00158143$.

6) Nuclear binding energy can be understood with a single energy coefficient of magnitude $\left(\frac{e_{s} e}{8 \pi \varepsilon_{0}\left(\hbar / m_{p} c\right)}\right) \cong 10.0 \mathrm{MeV}$.

7) In deuteron, there exists no strong interaction in between neutron and proton.

\section{Beta stability line with respect to strong coupling constant}

If $\alpha_{s} \cong 0.1186$, for $Z>8$, close to the line of beta stability,

$$
A_{s} \cong\left[Z+\left(\frac{e_{s}}{e}\right)\right]^{6 / 5} \cong(Z+2.904)^{1.2}
$$

See table 1, column-2.

For $Z>16$, close to the line of beta stability,

$$
\left.\begin{array}{l}
\left(A_{s}-2 Z\right) \cong(Z \beta+1)^{2}-4 \\
\text { where } \beta=\left(\frac{3}{5}\right) \alpha_{s} \cong 0.07116 .
\end{array}\right\}
$$

See table 1, column-3.

\section{Beta stability line with respect to nucleon mass difference}

With reference to nucleon and electron rest masses [1], we noticed that,

$$
\exp \left(\frac{\left(m_{n}-m_{p}\right) c^{2}}{m_{e} c^{2}}\right) \cong 12.5659102 \cong 4 \pi
$$

$\left\{\begin{array}{l}\text { where, } m_{n} c^{2} \cong 939.565413 \mathrm{MeV}, \\ m_{p} c^{2} \cong 938.272081 \mathrm{MeV} ; m_{e} c^{2} \cong 0.51099895 \mathrm{MeV}\end{array}\right.$
Based on this observation, beta stability line can be understood with the following empirical relations.

$$
\left.\begin{array}{l}
\text { Let, } k \cong(1 / 4 \pi)^{2} \cong 0.006333 \\
A_{s} \cong 2 Z+(Z / 4 \pi)^{2} \cong 2 Z+k Z^{2} \\
N_{s} \cong Z+(Z / 4 \pi)^{2} \cong Z+k Z^{2} \\
\left(Z / \sqrt{A_{s}-2 Z}\right) \cong 4 \pi
\end{array}\right\}
$$

\begin{tabular}{|c|c|c|c|}
\hline \multirow{2}{*}{$\begin{array}{c}\text { Proton } \\
\text { number } \\
\quad \boldsymbol{Z}\end{array}$} & \multicolumn{3}{|c|}{ Stable mass number $\boldsymbol{A}_{\boldsymbol{s}}$} \\
\hline & $\begin{array}{l}\text { Relation } \\
\text { (3) }\end{array}$ & $\begin{array}{l}\text { Relation } \\
\text { (4) }\end{array}$ & $\begin{array}{l}\text { Relation } \\
\text { (7) }\end{array}$ \\
\hline 2 & & & 4 \\
\hline 5 & & & 10 \\
\hline 8 & & & 16 \\
\hline 11 & 24 & & 23 \\
\hline 14 & 30 & 28 & 29 \\
\hline 17 & 36 & 35 & 36 \\
\hline 20 & 43 & 42 & 43 \\
\hline 23 & 50 & 49 & 49 \\
\hline 26 & 57 & 56 & 56 \\
\hline 29 & 64 & 63 & 63 \\
\hline 32 & 71 & 71 & 70 \\
\hline 35 & 78 & 78 & 78 \\
\hline 38 & 86 & 86 & 85 \\
\hline 41 & 94 & 93 & 93 \\
\hline 44 & 101 & 101 & 100 \\
\hline 47 & 109 & 109 & 108 \\
\hline 50 & 117 & 117 & 116 \\
\hline 53 & 125 & 125 & 124 \\
\hline 56 & 133 & 133 & 132 \\
\hline 59 & 141 & 141 & 140 \\
\hline 62 & 150 & 149 & 148 \\
\hline 65 & 158 & 158 & 157 \\
\hline 68 & 166 & 166 & 165 \\
\hline 71 & 175 & 175 & 174 \\
\hline 74 & 183 & 183 & 183 \\
\hline 77 & 192 & 192 & 192 \\
\hline 80 & 201 & 201 & 201 \\
\hline
\end{tabular}

See table 1, column-4. Based on these relations,
A) $\frac{\left(A_{s}-2 Z\right)^{2}}{A_{s}} \cong k^{2} A_{s} N_{s} \sqrt{Z}$
B) $\frac{A_{s}^{1 / 2} N_{s}^{1 / 4} Z^{1 / 8}}{\sqrt{A_{s}-2 Z}} \approx \frac{1}{\sqrt{k}} \approx 4 \pi$

Table-1: Estimated stable mass numbers 


\begin{tabular}{|c|c|c|c|}
83 & 209 & 210 & 210 \\
\hline 86 & 218 & 219 & 219 \\
\hline 89 & 227 & 228 & 228 \\
\hline 92 & 236 & 237 & 238 \\
\hline 95 & 245 & 246 & 247 \\
\hline 98 & 254 & 256 & 257 \\
\hline 101 & 263 & 265 & 267 \\
\hline 107 & 281 & 284 & 287 \\
\hline 110 & 291 & 294 & 297 \\
\hline 113 & 300 & 304 & 307 \\
\hline 116 & 309 & 314 & 317 \\
\hline
\end{tabular}

\section{Semi empirical relation for nuclear binding energy}

Based on the new integrated model proposed by N. Ghahramany et al $[12,13]$,

$$
B(Z, N)=\left\{A-\left(\frac{\left(N^{2}-Z^{2}\right)+\delta(N-Z)}{3 Z}+3\right)\right\} \frac{m_{n} c^{2}}{\gamma}
$$

where, $\gamma=$ Adjusting coefficient $\approx(90$ to 100$)$. if $N \neq Z, \delta(N-Z)=0$ and if $N=Z, \delta(N-Z)=1$.

We noticed that,

$$
\left.\begin{array}{l}
\frac{m_{n} c^{2}}{\gamma} \cong \frac{m_{n} c^{2}}{(90 \text { to } 100)} \\
\cong\left(\frac{e_{s} e}{8 \pi \varepsilon_{0}\left(\hbar / m_{p} c\right)}\right) \cong 10.0 \mathrm{MeV}
\end{array}\right\}
$$

And with reference to relation (7), it is also possible to show that, for $Z \cong(40$ to 83$)$, close to the beta stability line [7],

$$
\left[\frac{N_{s}^{2}-Z^{2}}{Z}\right] \cong k A_{s} Z
$$

Based on the above relations and proposed concepts, and with reference to the first four terms of the semi empirical mass formula, close to the beta stability line [8], if $\alpha_{s} \approx 0.1186$ and $R_{0} \approx 1.22 \mathrm{fm}$, semi empirically, we developed the following relations. a) Starting from $Z=3$, close to the beta stability line,

$$
\begin{aligned}
(B)_{A_{s}} & \left.\approx\left\{(Z-1)\left(\frac{e_{s} e}{4 \pi \varepsilon_{0}\left(\hbar / m_{p} c\right)}\right)\right\} \mp 10.0 \mathrm{MeV}\right\} \\
& \approx\{(Z-1) \times 20.0 \mathrm{MeV}\} \mp 10.0 \mathrm{MeV}
\end{aligned}
$$

For example, binding energy of Oxygen $(\mathrm{O})$ close to its stable atomic nuclides can be estimated to be $[(8-1) \times 20] \mp 10.0 \mathrm{MeV} \cong(130$ to 150$) \mathrm{MeV}$.

Binding energy of Iron (Fe) close to its stable atomic nuclides can be estimated to be $[(26-1) \times 20] \mp 10.0 \mathrm{MeV} \cong(490$ to 510$) \mathrm{MeV}$.

Binding energy of Tin (Sn) close to its stable atomic nuclides can be estimated to be $[(50-1) \times 20] \mp 10.0 \mathrm{MeV} \cong(970$ to 990$) \mathrm{MeV}$.

Binding energy of Lead $(\mathrm{Pb})$ close to its stable atomic nuclides can be estimated to be $[(82-1) \times 20] \mp 10.0 \mathrm{MeV} \cong(1610$ to 1630$) \mathrm{MeV}$.

b) For $A \approx(4$ to 80$)$, close to the beta stability line,

$$
\begin{aligned}
(B)_{A} & \left.\approx\left\{(A-\sqrt{A})\left(\frac{e_{s} e}{8 \pi \varepsilon_{0}\left(\hbar / m_{p} c\right)}\right)\right\}\right\} \\
& \approx\{(A-\sqrt{A}) \times 10.0 \mathrm{MeV}\}
\end{aligned}
$$

Binding energy of Helium- 4 can be approximately estimated to be $(4-\sqrt{4}) \times 10.0 \mathrm{MeV} \approx 20.0 \mathrm{MeV}$.

Actual binding energy is $28.296 \mathrm{MeV}$.

Binding energy of Carbon-12 can be approximately estimated to be $(12-\sqrt{12}) \times 10.0 \mathrm{MeV} \approx 95.5 \mathrm{MeV}$.

Actual binding energy is $92.162 \mathrm{MeV}$.

Binding energy of Scandium-45 can be approximately estimated to be $(45-\sqrt{45}) \times 10.0 \mathrm{MeV} \approx 382.92 \mathrm{MeV}$. Actual binding energy is $387.848 \mathrm{MeV}$. 
Binding energy of Manganese-55 approximately can be estimated to be, $(55-\sqrt{55}) \times 10.0 \mathrm{MeV} \approx 475.84 \mathrm{MeV}$. Actual binding energy is $482.072 \mathrm{MeV}$.

Binding energy of Kripton- 80 approximately can be estimated to be, $(80-\sqrt{80}) \times 10.0 \mathrm{MeV} \approx 710.56 \mathrm{MeV}$. Actual binding energy is $695.434 \mathrm{MeV}$.

c) For $(Z \geq 4)$, close to the beta stability line,

$$
\left.\begin{array}{l}
B_{(Z, A)} \cong\left\{A-\left[\left(\frac{k A Z}{2.531}+2.531\right)+1\right]\right\} 10.06 \mathrm{MeV} \\
\text { where }\left(\frac{\left(m_{n}-m_{p}\right) c^{2}}{m_{e} c^{2}}\right) \cong \ln (4 \pi) \cong 2.531 \text { and } \\
\left(\frac{k A Z}{2.531}+2.531\right)=\text { "New term" needs explanation }
\end{array}\right\}
$$

We are working on understanding the physical significance of $\left(\frac{k A Z}{2.531}+2.531\right)$. It needs further study at basic level.

See the following figure-1. Green curve represents the binding energy per nucleon estimated with the first four terms of SEMF relation (1) and (7). Dashed red curve represents the binding energy per nucleon estimated with relations (7) and (14).

Figure 1: Comparison of estimated and SEMF binding energy per nucleon

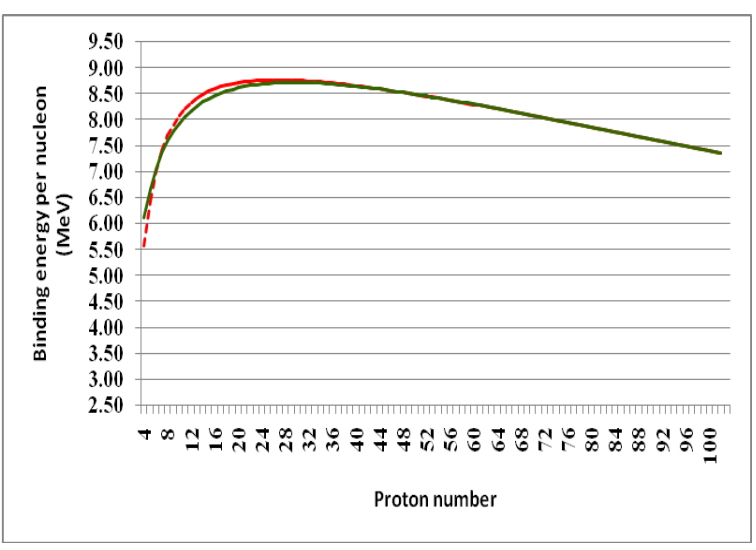

See table- 2 for the isotopic binding energy of $Z=50$

Table-2: Comparison of estimated and actual binding energy of isotopes of $\mathrm{Z}=50$

\begin{tabular}{|c|c|c|c|}
\hline $\begin{array}{c}\text { Mass } \\
\text { number } \\
A\end{array}$ & $\begin{array}{c}\text { Estimated } \\
\text { binding } \\
\text { energy } \\
(\mathrm{MeV}) \\
\text { Relation(14) }\end{array}$ & $\begin{array}{c}\text { Actual } \\
\text { binding } \\
\text { energy } \\
(\mathrm{MeV})\end{array}$ & $\begin{array}{c}\text { Error } \\
(\mathrm{MeV})\end{array}$ \\
\hline 112 & 950.2 & 953.532 & 3.3 \\
\hline 114 & 967.8 & 971.574 & 3.7 \\
\hline 115 & 976.6 & 979.121 & 2.5 \\
\hline 116 & 985.4 & 988.684 & 3.2 \\
\hline 117 & 994.2 & 995.627 & 1.4 \\
\hline 118 & 1003.0 & 1004.955 & 1.9 \\
\hline 119 & 1011.8 & 1011.438 & -0.4 \\
\hline 120 & 1020.6 & 1020.546 & -0.1 \\
\hline 122 & 1038.2 & 1035.53 & -2.7 \\
\hline 124 & 1055.9 & 1049.963 & -5.9 \\
\hline
\end{tabular}

\section{To understand the binding energies of Deuteron, Triton and ${ }_{2}^{4} \mathrm{He}$.}

If it is assumed that there exists no strong interaction in between neutron and proton, above relation (13) can be expressed as follows.

$$
\begin{aligned}
(B)_{A} & \left.\approx\left\{(A-\sqrt{A})\left(\frac{e^{2}}{8 \pi \varepsilon_{0}\left(\hbar / m_{p} c\right)}\right)\right\}\right\} \\
& \approx\{(A-\sqrt{A}) \times 3.443 \mathrm{MeV}\}
\end{aligned}
$$

Based on this relation (15), Deuteron $\left({ }_{1}^{2} H\right)$ binding energy can be estimated to be $2.02 \mathrm{MeV}$ and actual binding energy is $2.225 \mathrm{MeV}$.

From relation (15), Triton $\left({ }_{1}^{3} H\right)$ binding energy can be estimated to be $4.37 \mathrm{MeV}$. From relation (13), Triton $\left({ }_{1}^{3} H\right)$ binding energy can be estimated to be $12.68 \mathrm{MeV}$. Actual binding energy $(8.482 \mathrm{MeV})$ seems to be close to the average of (4.37 and 12.68) $\mathrm{MeV}=8.525 \mathrm{MeV}$. Clearly speaking, binding energy of $\left({ }_{1}^{3} H\right)$ seems to follow electromagnetic interaction as well as strong interaction and needs further study. 
In the similar way, $\left({ }_{2}^{3} \mathrm{He}\right)$ binding energy can be understood in terms of the combined effect of electromagnetic and strong interactions.

\section{Conclusion}

Nowadays, estimating and understanding nuclear binding energy with 'strong interaction' seems to attract many nuclear physicists. In this context, by considering the proposed semi empirical relations, existence of the 'strong elementary charge' can be confirmed. With further research, a realistic nuclear model pertaining to strong interaction can be developed.

\section{Acknowledgements}

Author Seshavatharam is indebted to his brother B.Vamsi Krishna for his valuable support.

\section{References}

[1] C. Patrignani et al. (Particle Data Group), Chin. Phys. C, 40, 100001 (2016) and 2017 update

[2] Weizsäcker, Carl Friedrich von, On the theory of nuclear masses; Journal of Physics 96 (1935) pages 431- 458.

[3] W. D. Myers et al. Table of Nuclear Masses according to the 1994 Thomas-Fermi Model.(from nsdssd.lbl.gov)

[4] P. Roy Chowdhury et al. Modified BetheWeizsacker mass formula with isotonic shift and new driplines. Mod.Phys.Lett. A20 (2005) 1605 1618.

[5] J.A. Maruhn et al., Simple Models of ManyFermion Systems, Springer-Verlag Berlin Heidelberg 2010. Chapter 2, page:45-70.

[6] Seshavatharam U. V. S, Lakshminarayana, S., A Virtual Model of Microscopic Quantum Gravity. Prespacetime Journal, Volume 9, Issue 1, pp. 58-82 (2018)

[7] U. V. S. Seshavatharam, Lakshminarayana S. Is 'reciprocal' of the strong coupling constant - a representation of upper limit of strength of nuclear binding energy? Submitted to QNP2018 conference, Nov.13-17, 2018, Tsukuba, Japan.

[8] U. V. S. Seshavatharam, Lakshminarayana S. On the possible existence of strong elementary charge and its applications. Submitted to ICNPAP-2018 conference, October 23-26, 2018, Centre for Applied Physics, Central University of Jharkhand, Ranchi, India.

[9] Seshavatharam U. V. S, Lakshminarayana, S., On the role of strong coupling constant and nucleons in understanding nuclear stability and binding energy. Journal of Nuclear Sciences, Vol. 4, No.1, 7-18, (2017)

[10] Seshavatharam U. V. S, Lakshminarayana, S., A Review on Nuclear Binding Energy Connected with Strong Interaction. Prespacetime Journal, Volume 8, Issue 10, pp. 1255-1271 (2018)

[11] U. V. S. Seshavatharam, Lakshminarayana S. To unite nuclear and sub-nuclear strong interactions. International Journal of Physical Research, 5 (2) 104-108 (2017)

[12] Ghahramany et al. New approach to nuclear binding energy in integrated nuclear model. Journal of Theoretical and Applied Physics 2012, 6:3

[13] N. Ghahramany et al. Stability and Mass Parabola in Integrated Nuclear Model. Universal Journal of Physics and Application 1(1): 18-25, (2013). 